Article

\title{
Simultaneous Prevention of Rotational and Translational Slip for a Humanoid Robot
}

\author{
Qinqin Zhou ${ }^{1}$ (D), Zhangguo Yu ${ }^{1,2, *}$, Si Zhang ${ }^{3}$, Xuechao Chen ${ }^{1,4}$, Mingyue Qin ${ }^{1}$, \\ Weimin Zhang ${ }^{1,2}$ and Qiang Huang ${ }^{1,2}$ \\ 1 Intelligent Robotics Institute, School of Mechatronical Engineering, Beijing Institute of Technology, \\ Beijing 100081, China; zhouqinqin@bit.edu.cn (Q.Z.); chenxuechao@bit.edu.cn (X.C.); \\ 2120170185@bit.edu.cn (M.Q.); zhwm@bit.edu.cn (W.Z.); qhuang@bit.edu.cn (Q.H.) \\ 2 Beijing Advanced Innovation Center for Intelligent Robots and Systems, Beijing 100081, China \\ 3 The High School Affiliated to Renmin University of China, Beijing 100080, China; zhangsi@rdfz.cn \\ 4 Key Laboratory of Biomimetic Robots and Systems, Ministry of Education, Beijing 100081, China \\ * Correspondence: yuzg@bit.edu.cn; Tel.: +86-010-6891-8230
}

Received: 20 August 2018; Accepted: 3 September 2018; Published: 4 September 2018

\begin{abstract}
Slip often occurs in humanoid robot walking, especially when the robot walks on a low friction floor or walks fast. Unexpected slip may cause the robot to fall and then sustain damage. In real environments, rotational and translational slip phenomena can happen during biped walking. Previous studies have mainly focused on solving these problems independently. In this paper, we propose strategies for simultaneous rotational and translational slip prevention based on a three mass model, which takes into account the effect of the swing leg. The rotational slip is prevented through a bionic walking pattern generator which mimics the yaw moment compensation mechanism of a human. The translational slip is eliminated through a novel reaction force ratio reduction control with the compensation of CoM (center of mass) acceleration. The effectiveness of the presented strategies is validated by simulations and experiments with an actual humanoid robot.
\end{abstract}

Keywords: humanoid robot; rotational slip prevention; translational slip prevention

\section{Introduction}

Encouraging achievements for humanoid robots, such as stable walking pattern generation [1-3], dexterous mechanical design [4-6], and adaptive performance improvement [7-9], have been made during the last few decades. However, numerous problems concerned with humanoid robots operating in natural environments reliably remain unsolved. One of these problems consists in the prevention of slip during biped walking. Slip may cause humanoid robots to walk unstably or even fall. Therefore, slip prevention is a crucial issue for real-world applications of humanoid robots.

Previous studies address that the slip of a humanoid robot during walking is divided into rotational slip and translational slip [10]. Thus, two categories of slip prevention strategies have emerged.

On the aspect of rotational slip, many studies focus on the important effects between inertial moments and friction moments. They prevent rotational slipping by balancing the inertial and the friction moments with controlling the movements of the upper body [11-14]. A class of methods reduced the inertial moments by the arm swing strategy [11,12]. Collins et al. proposed that the arm swing effectively prevented slipping during biped walking since it reduced vertical ground reaction moment by $63 \%$ [11]. Punt et al. found that excessive arm swing in comparison to normal arm swing prevented rotational slipping and increased the biped walking stability dramatically [12]. Another class of methods compensated the inertial moments in yaw to prevent support foot slip as the upper 
body movements strategy $[13,14]$. Ugurlu et al. decreased the yaw moment by rotating the upper body, which created a secondary moment to counteract the undesired moment [13]. Cisneros, et al. presented a generalized approach for compensating the required yaw moment of the humanoid robot by modifying the motion of the waist joint in order to prevent rotational slipping [14]. Although the above strategies demonstrate the validity of handling the inertial moments to prevent rotational slipping, the calculations for upper body movements are relatively complicated since they are computed from complex robot models.

On the aspect of translational slip prevention, most of the studies concentrate on the frictional constraint between the sole of the humanoid robot and the floor. Moreover, they prevent translational slipping by causing the friction which is required for humanoid robot to walk less than the maximum friction that the ground can provide [15-20]. Khadiv et al. and Brandao et al. developed a gait optimization routine to generate walking patterns. These walking patterns demanded the lowest friction forces for implementation in order to prevent translational slipping $[15,16]$. Vázquez et al. and Hashlamon et al. designed sensors as a measurement system of slip and then adapted the contact points of the robot with the walking surface in order to increase the frictional characteristics $[17,18]$. Takabayashi et al. and Bowyer et al. calculated the frictional force to deduce the quantitative expression of frictional constraint. Constraining the friction of fast walking within the computed constraint could then prevent translational slipping during fast walking $[19,20]$. The above methods handled the translational slip phenomenon by reducing the required friction for biped walking or enhancing the friction between the sole of the robot and the ground. However, these methods only consider translational slip, without considering rotational slip, which is important in actual biped walking.

In fact, the rotational and translational slip occur at the same time in biped walking, especially in fast walking $[19,21]$. Most of the researchers propose methods that only target one of these types of slip. Very few works discuss how to prevent rotational and translational slip simultaneously [11-15,17,19]. Furthermore, most of these previous strategies, such as inverted pendulum model or inverted pendulum flywheel model [12-14,19], utilize the simplified model. In these models, a humanoid robot is regarded as a mass point so that the influences of the swing leg are ignored. However, the effect of the swing leg is a significant matter in slip prevention.

In order to overcome the above deficiencies, this paper presents a simultaneous prevention for rotational and translational slip during humanoid walking. The main contributions are as follows:

(1) A three mass model is utilized to improve the accuracy of the dynamic model for slip prevention. It takes into account the effect of swing leg on robot slip.

(2) A bionic walking pattern generator by mimicking the yaw moment compensation mechanism of humans is presented for rotational slip prevention. It effectively reduces the yaw moment with the optimized trajectories of swing arm and rotational waist based on the three mass model.

(3) A novel reaction force ratio reduction control using CoM (center of mass) acceleration compensation is proposed for translational slip prevention. It rapidly makes the ratio of tangential force to normal reaction force smaller than the coefficient of the ground friction.

The rest of the paper is organized as follows. Section 2 introduces the dynamic model of slip prevention. Section 3 analyzes the yaw moment compensation mechanism of human walking and then presents a human-inspired walking pattern generator for rotational slip prevention. Section 4 provides a novel reaction force ratio reduction controller for translational slip prevention. Section 5 demonstrates the effectiveness of the proposed slip prevention methods with simulations and experiments. Section 6 concludes the paper. 


\section{Dynamics Model of Slip Prevention}

\subsection{Slip Prevention Scheme Overview}

Slip usually appears quite suddenly and urgently. Humans manage to overcome slip in daily walking in a natural way through coordinated body movements. The arm swing and waist rotation play important roles in compensating the yaw moment in the vertical direction, and then prevent the possibility of potential rotational slip due to a reduction of the inertial moments. Therefore, we propose a bionic walking pattern generator to prevent rotational slipping by mimicking the yaw moment compensation mechanism of human.

Although the generated walking pattern can prevent the rotational slip, the translational slip still exists during biped walking. As described in the introduction, the translational slip is mainly caused by the fact that the ratio of tangential force to normal reaction force is greater than the coefficient of the ground friction. Therefore, we propose a novel reaction force ratio reduction controller to prevent translational slipping by quickly compensating the CoM acceleration.

Figure 1 shows the overview of the control architecture for translational and rotational slip prevention. First, according to the human walking mechanism, the rotational prevention pattern generator produce the desired walking pattern, which eliminates the rotational slip. The reference CoM acceleration, $\left(\ddot{x}_{c}, \ddot{z}_{c}\right)$, is obtained. However, this walking pattern cannot make humanoid robots walk stably because of the translational slip. The translational slip prevention controller thus works based on the current robot state, which computes the compensation of CoM acceleration $\left(\Delta \ddot{x}_{c}, \Delta \ddot{z}_{c}\right)$. If the ratio of the tangential force to normal reaction force is within the designated range, the translational slip may not happen. The CoM acceleration compensation should be $\Delta \ddot{x}_{c}=0$ and $\Delta \ddot{z}_{c}=0$. Otherwise, at least one of the value of the CoM acceleration compensation is not equal to 0 . This CoM acceleration compensation is added to the reference CoM acceleration. The compensated CoM acceleration affects the Zero Moment Point (ZMP), and the modified ZMP then adjusts the joint angles. Finally, the humanoid robot can walk stably without rotational or translational slip.

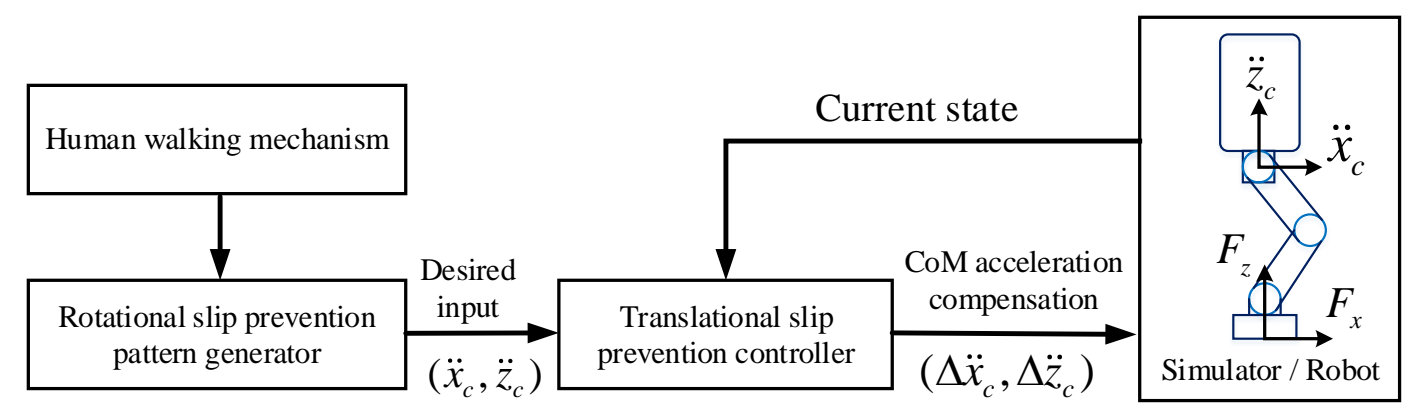

Figure 1. Overview of the control architecture for translational and rotational slip prevention.

\subsection{The Three Mass Model}

A humanoid robot is a highly non-linear and strong coupling system with complex dynamics and kinematic constraints. It is difficult to estimate the current state by this complicated model. In order to obtain the real-time state of the robot quickly, the model of the humanoid robot should be simplified. Meanwhile, as the introduction describes, the simplified model should also take the swing leg into consideration. Hence, we utilized a three mass model to analyze slip prevention.

The three mass model proposed in this paper consists of three point masses, as Figure 2 depicts. One of the point masses is at the end of the inverted pendulum, and the other two are at the ankle of the support leg and the swing leg. 
To analyze the rotational slip, the lateral plane is shown in Figure 2a. When the walking speed and the foot trajectories of the robot have been designated, the dynamics of the swing leg is derived. The yaw moment of the swing leg for the robot is calculated as

$$
M_{s w g}=m_{s w g} d_{h} \ddot{x}_{s w g}
$$

where $M_{\text {swg }}$ is the yaw moment of the swing leg; $m_{s w g}$ is the mass of the swing leg; $d_{h}$ is the distance between the projection point of the swing leg center on the ground and the projection point of CoM on the ground; $\ddot{x}_{s w g}$ is the acceleration of swing leg in $x$ direction.

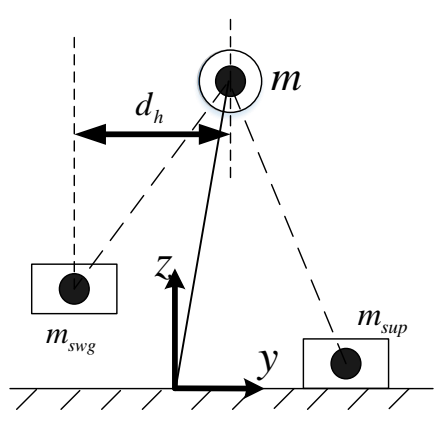

(a) Three mass model in lateral plane.

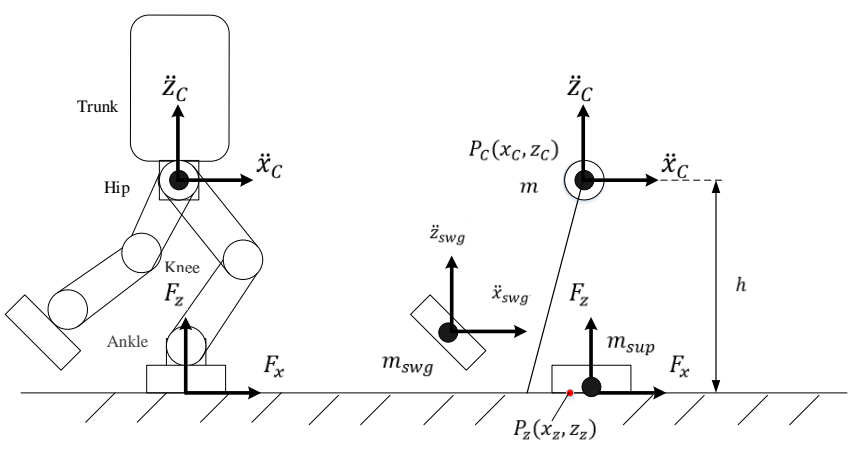

(b) Three mass model in sagittal plane.

Figure 2. Three mass model for humanoid robot.

Once the yaw moment of the swing leg is larger than the friction moment, the support leg of the humanoid robot may rotate on the ground and deviate the robot from its desired path. In order to prevent this rotational slipping, the yaw moment of the swing leg should be reduced, which is realized by a human-inspired walking pattern generator as the following section describes.

To study the translational slip, the sagittal plane is shown in Figure $2 \mathrm{~b} . P_{C}\left(x_{C}, z_{C}\right)$ is the position of CoM and $P_{z}\left(x_{z}, z_{z}\right)$ is the position of ZMP. The ground reaction force to the support leg is decomposed into two parts. One is horizontal and the other is vertical. The horizontal and vertical forces are computed as

$$
\begin{aligned}
& F_{x}=m \ddot{x}_{c}+m_{s w g} \ddot{x}_{s w g} \\
& F_{z}=m \ddot{z}_{c}+m g+m_{s w g}\left(g+\ddot{z}_{s w g}\right)+m_{\text {sup }} g
\end{aligned}
$$

where $F_{x}$ and $F_{z}$ are the horizontal and vertical reaction forces, respectively; $m, m_{\text {swg }}, m_{\text {sup }}$, and $m_{\text {total }}$ are the mass of inverted pendulum, the swing leg, the support leg, and the whole model, respectively; $\ddot{x}_{c}$ and $\ddot{z}_{c}$ are the horizontal and vertical acceleration of CoM, respectively; $\ddot{x}_{s w g}$ and $\ddot{z}_{s w g}$ are the horizontal and vertical acceleration of the swing leg, respectively; $g$ is the gravity acceleration; $h$ is the height of the CoM.

It is obvious that the humanoid robot may slip when the ratio of tangential force to normal reaction force is greater than the coefficient of the friction between the foot sole and the ground. The horizontal force, $F_{x}$, is a tangential force, while the vertical force, $F_{z}$, is a normal reaction force. In order to prevent translational slipping, the following equation should be satisfied:

$$
\left|F_{x} / F_{z}\right| \leq \mu_{0}
$$

where $\mu_{0}$ is the threshold of the coefficient of friction. The material of the foot sole and the ground is known, so the coefficient of static friction between the foot sole and the ground surface is estimated. Then, the threshold of the coefficient of friction, $\mu_{0}$, is set to be smaller than this estimated coefficient of static friction in order to handle the ground surface of this material. 
Then, by substituting Equation (2) into Equation (3), the acceleration constraint can be derived as

$$
\left|F_{x} / F_{z}\right|=\left|\left(a \ddot{x}_{c}+b \ddot{x}_{s w g}\right) /\left(a \ddot{z}_{c}+b \ddot{z}_{s w g}+g\right)\right| \leq \mu_{0}
$$

where $a=m / m_{\text {total }}$ and $b=m_{\text {swg }} / m_{\text {total }}=m_{\text {sup }} / m_{\text {total }}$.

Equation (4) indicates that the accelerations of CoM and swing leg are critical to restrict the horizontal and vertical reaction force. Therefore, Equation (4) is treated as a translational slip indicator since it can estimate the state of the humanoid robot. In order to prevent translational slipping, the accelerations of CoM and swing leg should be limited to Equation (4), which is achieved by a reaction force ratio reduction controller as the next section states.

\section{Rotational Slip Prevention}

A human-inspired walking pattern generator for humanoid robot is proposed to prevent rotational slipping in this section. Firstly, we reviewed the yaw moment compensation mechanism when human walks, which is summed up in our previous work [22]. The motion design and parameters optimization based on the three mass model is then presented, including the motion design of upper body and the reference trajectory of the whole body generation.

\subsection{Yaw Moment Compensation Mechanism for Human Walking}

The gait pattern of the human walking is recorded by the optical motion capture system, which consists of 12 optical cameras located around the ceiling board and 38 retro-reflective markers fixed on each human subject. Several of these markers are intentionally located on the shoulder and waist parts for the motion capture in this study. The cameras receive the returned signals from the markers, capture their varied positions, and thus record the gait pattern of the subjects, as Figure 3 shows. The frequency for the data update is 100 frames per second. The yaw moment between feet and ground in the human walking can be measured using the yaw moment measurement system [23].

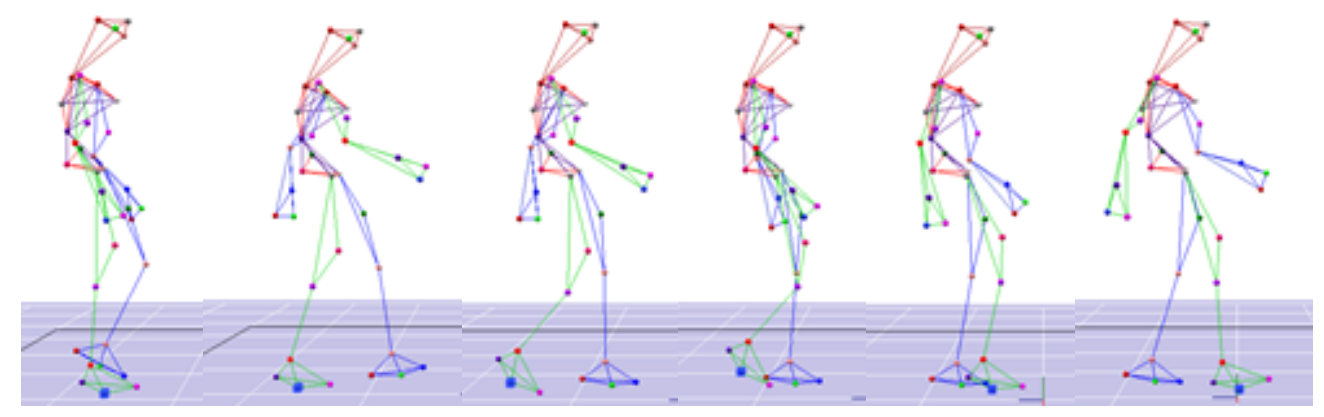

Figure 3. Snapshots of the human walking experiment by the motion capture system.

The gait pattern data are acquired from several subjects for universality, and each subject is required to walk at different speeds (ranging from 4 to $6 \mathrm{~km} / \mathrm{h}$ ) and walk with varied upper body motions (walk naturally with arm-swing and waist rotation, walk without waist rotation or arm-swing, and walk without arm-swing and with waist rotation). The details of the human walking data collection experiments are shown in [23]. Several important rules can be drawn after studying the yaw moment compensation mechanism in human walking [22].

- The arm swing and waist rotation in the vertical direction contribute to the yaw moment compensation in the walking.

- The arm swings in the back-and-forth pattern periodically, and the amplitude of the swing arm increases as the step length gets larger during the walking. 
- The second derivative of the angle trajectory of the swing arm shows that the acceleration of the swing angle undergoes roughly the five stages: ascent, light fluctuation, descent, light fluctuation, and ascent.

- The waist rotation in the vertical direction occurs in the similar form as the periodic sinusoidal signal in human walking, and the rotation angle becomes larger when the walking speed increases.

\subsection{Yaw Moment Compensation for Humanoid Robot Walking}

In order to prevent rotational slipping, the yaw moment compensation for humanoid robot walking is designed based on the yaw moment compensation mechanism of human walking introduced above. As the rules of human walking describe above, arm swing and waist rotation could reduce the yaw moment to a certain degree. Thus, the yaw moment compensation for humanoid robot walking consists of arm swing and waist rotation.

Since the arm swings in the back-and-forth pattern during human walking, the shoulder joint of humanoid robot that makes the arm swing in the pitch direction should rotate and other arm joints of humanoid robot should be fixed. According to the five stages of the arm swing described above, the acceleration of the shoulder joint $\ddot{\theta}_{a}(t)$ is designed as follows:

$$
\ddot{\theta}_{a}(t)= \begin{cases}k_{a} D_{\text {step }} a_{j} t, & 0 \leq t \leq T_{1} \\ k_{a} D_{\text {step }} a_{\text {max }}, & T_{1}<t \leq T-T_{1} \\ -k_{a} D_{\text {step }} a_{j}(t-T), & T-T_{1}<t \leq T+T_{1} \\ -k_{a} D_{\text {step }} a_{\text {max }}, & T+T_{1}<t \leq T_{f}-T_{1} \\ k_{a} D_{\text {step }} a_{j}\left(t-T_{f}\right), & T_{f}-T_{1}<t \leq T_{f}\end{cases}
$$

where $a_{j}$ is the maximum value of the jerk for the shoulder joint, which is determined by the actuators; $D_{\text {step }}$ is the step length for the designated walking speed; $k_{a}$ is the adjustable coefficient of the jerk for real application, which should satisfy $0 \leq k_{a} \leq 1 / D_{\text {step }}$; $a_{\max }$ is the maximum acceleration for the shoulder joint; $T_{1}$ is the predefined time for the acceleration increase or decrease which is derived from $T_{1}=a_{\max } / a_{j} ; T_{f}$ is the planning time for the motion design, which should satisfy $T_{f}=2 T$.

Then the three mass model is used to compute the constraint of yaw moment [24]. The approximated moment in the vertical direction of one single swing arm, $M_{a}(t)$, is given as

$$
M_{a}(t)=\frac{1}{2} L_{a} m_{a} \ddot{\theta}_{a}(t) W_{s}
$$

where $m_{a}$ is the mass of the swing arm; $L_{a}$ is the length of the swing arm; $W_{s}$ is the distance between the projection point of the swing arm center on the ground and the projection point of CoM on the ground.

Since the waist rotation is related to the walking speed according to the yaw moment compensation mechanism of human walking, the rotation angle of waist is designed as follows:

$$
\theta_{w}(t)=k_{w} v \sin \left(\frac{\pi t}{T_{\text {step }}}\right)
$$

where $\theta_{w}$ is the rotation angle of the waist; $k_{w}$ is the adjustable coefficient for real application which should satisfy $0 \leq k_{w} \leq \theta_{\max } / v ; v$ is the walking speed of humanoid robot; $T_{\text {step }}$ is the walking cycle time. $\theta_{\max }$ is the norm of the predefined boundary value of the waist rotation angle.

The approximated moment in the vertical direction of the waist, $M_{w}(t)$, is given as

$$
M_{w}=\left(I_{w}+m_{w} d_{w}^{2}\right) \ddot{\theta}_{w}(t)
$$


where $I_{w}$ is the moment of inertia of the waist; $d_{w}$ is the distance between the projection point of CoM on the ground and the center of the foot-ground contact area.

The rotational slip may be prevented unless the moments in the vertical direction of the swing arm and waist satisfy the following constraint:

$$
\begin{aligned}
& M_{w}+M_{a}+M_{s w g} \leq M_{f t \max } \\
& M_{w}\left(k_{w}\right)+M_{a}\left(k_{a}\right)+M_{s w g} \leq M_{f t \max }
\end{aligned}
$$

where $M_{f t \max }$ is the maximum frictional moment provided by the contact conditions, which can be estimated using the method in [10]. The constraints of the adjustable parameters $k_{a}$ and $k_{w}$ for rotational slip prevention is depicted as

$$
\left(k_{a}, k_{w}\right) \in \Omega
$$

where $\Omega$ is the area for the restricted values determined by $0 \leq k_{a} \leq 1,0 \leq k_{w} \leq \theta_{\max } / v$, and the constraints of Equation (9).

\subsection{Optimized Pattern Generation for Rotational Slip Prevention}

In order to generate a gait pattern for stable biped walking, parameters for the hip and feet in the sagittal plane should be optimized. In this study, we optimize the gait pattern by mimicking the yaw moment compensation mechanism of human walking, taking full account of the contributions of the arm swinging and waist rotation in the yaw moment compensation based on the three mass model.

Figure 4 illustrates the process of the gait pattern generation, where the optimized parameters of arm swing and waist rotation are $k_{a}$ and $k_{w}$. After the foot parameters are determined considering the walking speed and the ground conditions, the hip positions $x_{h}$ and $z_{h}$ are used for ZMP calculation to select the gait pattern that produces the maximum stability margin (the method for selecting hip position is proposed in [8]).

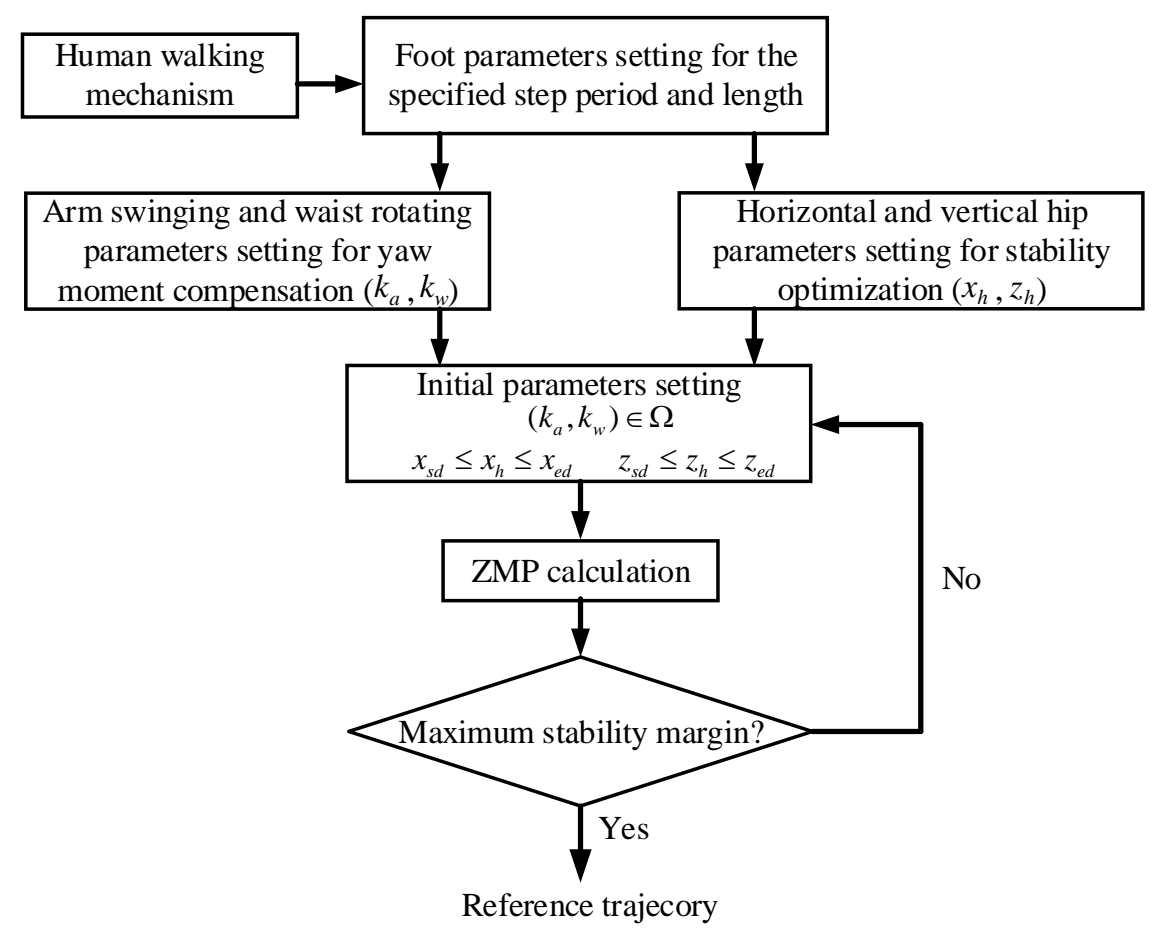

Figure 4. Flowchart of the optimized gait pattern generation. 


\section{Translational Slip Prevention}

A reaction force ratio reduction controller for humanoid robots is proposed to prevent translational slipping. The flow chart of this translational slip prevention controller is shown in Figure 5. This controller computes the compensation of CoM acceleration and then adds the compensation to the reference CoM acceleration. The CoM acceleration is associated with the ZMP. When the humanoid robot tends to slip, the ZMP is modified according to the CoM acceleration compensation, which means the ratio of the reaction force is modified. As long as the ratio of the reaction force is reduced according to the compensation, the translational slip prevention is achieved.

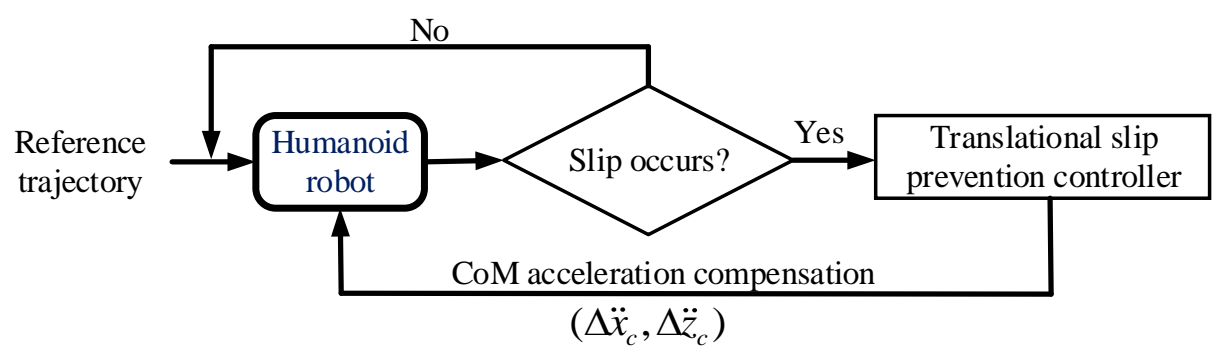

Figure 5. Flowchart of the translational slip controller.

\subsection{ZMP Deviation}

According to the definition of ZMP in [25], the ZMP in the $x$ direction, $x_{z}$ can be calculated as

$$
x_{z}=\left[\sum_{i=0}^{n} m_{i}\left(\ddot{z}_{i}+g\right) x_{i}-\sum_{i=0}^{n} m_{i} \ddot{x}_{i} z_{i}-\sum_{i=0}^{n} I_{i y} \ddot{\Omega}_{i y}\right] / \sum_{i=0}^{n} m_{i}\left(\ddot{z}_{i}+g\right)
$$

where $n$ is the number of links; $m_{i}$ is the mass of link $i ;\left(x_{i}, y_{i}, z_{i}\right)$ is the coordinate of the mass center of link $i ; I_{i}$ is the rotational inertial of link $i ; \ddot{\Omega}_{i}$ is the angular acceleration of link $i$.

In order to simplify calculations, the items $\sum_{i=0}^{n} I_{i y} \ddot{\Omega}_{i y}$ are ignored. Since a three mass model is utilized, the ZMP in the $x$ direction as Equation (11) shows can be rewritten as

$$
\begin{aligned}
x_{z}= & {\left[m\left(\ddot{z}_{c}+g\right) x_{c}-m \ddot{x}_{c} z_{c}+m_{s w g}\left(\ddot{z}_{s w g}+g\right) x_{s w g}-m_{s w g} \ddot{x}_{s w g} z_{s w g}\right] } \\
& /\left[m\left(\ddot{z}_{c}+g\right)+m_{s} w g\left(\ddot{z}_{s w g}+g\right)+m_{s u p g}\right] \\
= & {\left[a\left(\ddot{z}_{c}+g\right) x_{c}-a \ddot{x}_{c} z_{c}+b\left(\ddot{z}_{s w g}+g\right) x_{s w g}-b \ddot{x}_{s w g} z_{s w g}\right] } \\
& /\left(g+a \ddot{z}_{c}+b \ddot{z}_{s w g}\right) .
\end{aligned}
$$

Here, we assume that $\Delta t$ is the time interval and that $\Delta x_{z}$ is the ZMP deviation. Equation (12) is discretized by the time interval, and the equation at the current time $t(k)(k=0,1,2 \ldots N)$ is then shown as:

$$
\begin{aligned}
x_{z}= & {\left[a\left(\ddot{z}_{c}(k)+g\right) x_{c}(k)-a \ddot{x}_{c}(k) z_{c}(k)+b\left(\ddot{z}_{s w g}(k)+g\right) x_{s w g}(k)-b \ddot{x}_{s w g}(k) z_{s w g}(k)\right] } \\
& /\left[g+a \ddot{z}_{c}(k)+b \ddot{z}_{s w g}(k)\right] .
\end{aligned}
$$

The dynamics balance equation at the next control period $t(k+1)$ is derived as

$$
\begin{aligned}
x_{z}= & {\left[a\left(\ddot{z}_{c}(k+1)+g\right) x_{c}(k+1)-a \ddot{x}_{c}(k+1) z_{c}(k+1)+b\left(\ddot{z}_{s w g}(k+1)+g\right) x_{s w g}(k+1)\right.} \\
& \left.-b \ddot{x}_{s w g}(k+1) z_{s w g}(k+1)\right] /\left[g+a \ddot{z}_{c}(k+1)+b \ddot{z}_{s w g}(k+1)\right] .
\end{aligned}
$$


After the extremely short time interval $\Delta t$, the accelerations, CoM, and ZMP positions at the next control period can be obtained through the Taylor formula. Ignoring the quadratic and higher order terms of $\Delta t$, the relation between each item after the short-term increment $\Delta t$ is computed as

$$
\begin{gathered}
\ddot{x}_{c}(k+1)=\ddot{x}_{c}(k)+\Delta \ddot{x}_{c}(k), \quad \ddot{x}_{s w g}(k+1)=\ddot{x}_{s w g}(k)+\Delta \ddot{x}_{s w g}(k), \quad \ddot{z}_{c}(k+1)=\ddot{z}_{c}(k)+\Delta \ddot{z}_{c}(k) \\
z_{c}(k+1)=z_{c}(k)+\Delta z_{c}(k), \quad z_{s w g}(k+1)=z_{s w g}(k)+\Delta z_{s w g}(k), \quad x_{z}(k+1)=x_{z}(k)+\Delta x_{z}(k) \\
x_{c}(k+1)=x_{c}(k)+\Delta x_{c}(k), \quad \ddot{z}_{s w g}(k+1)=\ddot{z}_{s w g}(k)+\Delta \ddot{z}_{s w g}(k), \quad x_{s w g}(k+1)=x_{s w g}(k)+\Delta x_{s w g}(k) .
\end{gathered}
$$

Then, substituting Equations (13) and (14) into Equation (15), the dynamics balance equations in the adjacent control periods is obtained as

$$
\begin{aligned}
& \Delta x_{z}(k)\left[g+a \ddot{z}_{c}(k)+b \ddot{z}_{s w g}(k)\right]+a \dot{x}_{c}(k) \Delta t\left[x_{z}(k)+\Delta x_{z}(k)\right]\left[a \Delta \ddot{z}_{c}(k)+b \Delta \ddot{z}_{s w g}(k)\right] \\
& =a\left[\ddot{z}_{c}(k)+g\right] \dot{x}_{c}(k) \Delta t+a \Delta \ddot{z}_{c}(k)\left[x_{c}(k)+\dot{x}_{c}(k) \Delta t\right]-a\left[\ddot{x}_{c}(k) \dot{z}_{c}(k) \Delta t+\Delta \ddot{x}_{c}(k) z_{c}(k)\right. \\
& \left.+\ddot{x}_{c}(k) \dot{x}_{c}(k) \Delta t\right]+b \dot{x}_{s w g}(k) \Delta t\left[\ddot{z}_{s w g}(k)+g\right]+b \Delta \ddot{z}_{c}(k)\left[x_{s w g}(k)+\dot{x}_{s w g}(k) \Delta t\right] \\
& -b\left[s \ddot{w} g_{c}(k) \dot{z}_{c}(k) \Delta t+\Delta s \ddot{w} g_{c}(k) z_{c}(k)+s \ddot{w} g_{c}(k) \dot{x}_{c}(k) \Delta t\right] .
\end{aligned}
$$

When ignoring the items $\Delta \ddot{z}_{c}(k), \Delta x_{z}(k), \Delta \ddot{z}_{s w g}(k)$, and $\Delta x_{z}(k)$, the relationship between the increments of the accelerations and the ZMP is expressed as

$$
\begin{aligned}
& a\left(x_{z}-x_{c}-\dot{x}_{c} \Delta t\right) \Delta \ddot{z}_{c}+b\left(x_{z}-x_{s w g}-\dot{x}_{s w g} \Delta t\right) \Delta \ddot{z}_{s w g}+a\left(z_{c}+\dot{z}_{c} \Delta t\right) \Delta \ddot{x}_{c}+b\left(z_{c}+\dot{z}_{s w g} \Delta t\right) \Delta \ddot{x}_{s w g} \\
& =a\left(\ddot{z}_{c}+g\right) \dot{x}_{c} \Delta t-a \ddot{x}_{c} \dot{z}_{c} \Delta t+b\left(\ddot{z}_{s w g}+g\right) \dot{x}_{s w g} \Delta t-b \ddot{x}_{s w g} \dot{z}_{s w g} \Delta t-\left(g+a \ddot{z}_{c}+b \ddot{z}_{s w g}\right) \Delta x_{z} .
\end{aligned}
$$

\subsection{Translational Slip Prevention Control}

When the ratio of tangential force to normal reaction force is greater than the coefficient of friction, the humanoid robot may slip. In order to prevent this translational slipping, the ratio should be reduced by controlling the acceleration of CoM and swing leg. However, the acceleration of swing leg mainly causes rotational slip, which has been eliminated by the above human-inspired walking pattern generator. Hence, the translational slip caused by the acceleration of swing leg is negligible. Therefore, only the CoM acceleration is concerned.

According to the above, a novel CoM acceleration compensation controller is designed. The CoM acceleration compensation consists of two parts. One is vertical and the other is horizontal. Compared with the compensation of horizontal CoM acceleration, the compensation of vertical CoM acceleration has less effect on ZMP, which determines the stable walking of a humanoid robot. Thus, the vertical CoM acceleration is compensated first. If the translational slip still occurs after compensating the vertical CoM acceleration, the horizontal CoM acceleration will be compensated.

The acceleration of swing leg is ignored, so $\Delta \ddot{z}_{s w g}$ and $\Delta \ddot{x}_{s w g}$ is treated as 0 . The compensation of vertical CoM acceleration is first designed to produce a desired ZMP increment $\Delta x_{z}$, as follows:

$$
\Delta \ddot{z}_{c}= \begin{cases}k /\left[a\left(x_{z}-x_{c}-\dot{x}_{c} v \Delta t\right)\right], & \left|F_{x} / F_{z}\right| \leq \mu_{0} \\ F_{z} / m-g / a-b \ddot{z}_{s w g} / a, & \left|F_{x} / F_{z}\right| \geq \mu_{0}\end{cases}
$$

where $k=a\left(\ddot{z}_{c}+g\right) \dot{x}_{c} \Delta t-a \ddot{x}_{c} \dot{z}_{c} \Delta t+b\left(\ddot{z}_{s w g}+g\right) \dot{x}_{s w g} \Delta t-b \ddot{x}_{s w g} \dot{z}_{s w g} \Delta t-\left(g+a \ddot{z}_{c}+b \ddot{z}_{s w g}\right) \Delta x_{z}$. In this situation, $\Delta \ddot{x}_{c}$ is considered to be 0. If $\Delta \ddot{z}_{c}$ satisfies the constraint of Equation (4), then the CoM acceleration compensation control is finished. 
However, if $\Delta \ddot{z}_{c}$ cannot satisfy the constraint of Equation (4), the actual $\Delta \ddot{z}_{c}$, which produces $\mu_{0}$, will be adopted. Meanwhile, the horizontal CoM acceleration is compensated. After $\Delta \ddot{z}_{c}$ has been determined, the compensation of horizontal CoM acceleration is determined as

$$
\Delta \ddot{x}_{c}=\left\{\begin{array}{ll}
{\left[k-a\left(x_{z}-x_{c}-\dot{x}_{c} v \Delta t\right)\right] /\left[a\left(z_{c}+\dot{z}_{c} \Delta t\right)\right],} & \left|F_{x} / F_{z}\right| \leq \mu_{0} \\
\left(g+a \ddot{z}_{c}+b \ddot{z}_{s w g}\right) \mu_{0}-b \ddot{x}_{s w g} / a-b \ddot{x}_{c} / a, & F_{x} / F_{z}>\mu_{0} \\
-\left(g+a \ddot{z}_{c}+b \ddot{z}_{s w g}\right) \mu_{0}-b \ddot{x}_{s w g} / a-b \ddot{x}_{c} / a, & F_{x} / F_{z}<-\mu_{0}
\end{array} .\right.
$$

The vertical CoM acceleration is compensated at first, as Equation (18) shows which has less influence on ZMP. If the constraint of Equation (4) is still unsatisfied, the horizontal CoM acceleration is compensated. Meanwhile, the magnitude of CoM acceleration compensation is limited in actual experiments. Therefore, this CoM acceleration compensation control is able to prevent the translational slip while preserving the ZMP in the support polygon.

\section{Simulations and Experiments}

To validate the effectiveness of the proposed methods for slip prevention, simulations and experiments are tested on our BHR- 5 humanoid robot. Physical parameters of BHR-5 are listed in [8]. The walking speed is $1.5 \mathrm{~km} / \mathrm{h}$ with the length of $0.6 \mathrm{~m}$ and the step period of $1.4 \mathrm{~s}$.

\subsection{Simulations}

The physical parameters of the virtual biped robot model are the same as the actual robot. The robot walked on a floor in the simulations. The physical parameters of the ground is set according to the actual ground in the experiments. The coefficient of static friction is estimated to be 0.5 . Then, the threshold of the coefficient of friction, $\mu_{0}$, is set to be 0.3 .

The comparison of without and with proposed slip prevention control is shown in Figure 6. The robot is commanded to walk along the straight dotted lines. Figure 6a shows that, without the slip prevention control proposed in this paper, the rotation slip occurs since the swing leg of the fast walking robot brings too large a yaw moment. Hence, the robot cannot walk along the dotted line. When the proposed slip prevention control is applied, this slip is prevented successfully, and the robot can walk along the dotted line, as Figure $6 \mathrm{~b}$ shows.

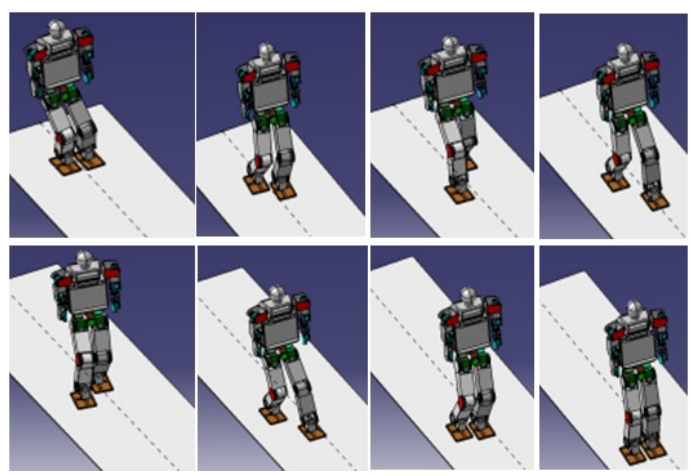

(a) Walking simulation without slip prevention method.

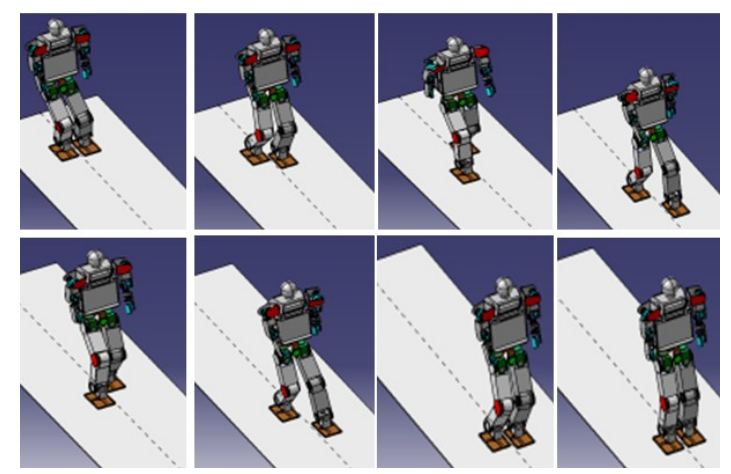

(b) Walking simulation with slip prevention method.

Figure 6. Humanoid robot walking simulations.

Figure 7 shows the yaw moment with and without rotational prevention control during biped walking in simulation. As the blue line depicts, the robot would suffer from large rotational slip without the proposed method since the yaw moment has large fluctuations because of the huge moment of the swing leg. When the proposed human-inspired walking pattern generation is utilized, the arm swinging and waist rotation effectively reduce the yaw moment as the red line depicts. 
Thus, the proposed rotational slip prevention control, which generates the walking pattern based on the yaw moment compensation mechanism in humans, is effective.

Figure 8a shows the ratio of the horizontal reaction force to the vertical reaction force, which is $F_{x} / F_{z}$. As Equation (3) indicates, if the ratio of horizontal force to vertical force is smaller than the coefficient of the ground friction, the possibility of translational slip is smaller. When the proposed CoM acceleration compensation control works as the red line shows, the reaction force ratio is smaller than that without control which is plotted in blue line. That means the proposed CoM acceleration compensation control prevents the translational slip to a certain degree.

Meanwhile, Figure $8 \mathrm{~b}$ compares the ZMP in the forward direction with and without translational slip prevention control during biped walking. The original ZMP as the blue line depicts, is produced by the walking planning method in [8]. Thus, this ZMP is modified according to the CoM acceleration compensation and is depicted in the red line. Both ZMP are within the maximum stability margin. However, the red line is more close to the boundary of the stability margin, which means that the proposed method sacrifices part of the ZMP based on stability for slip prevention. This is realistic and quite helpful for humanoid walking in real environments.

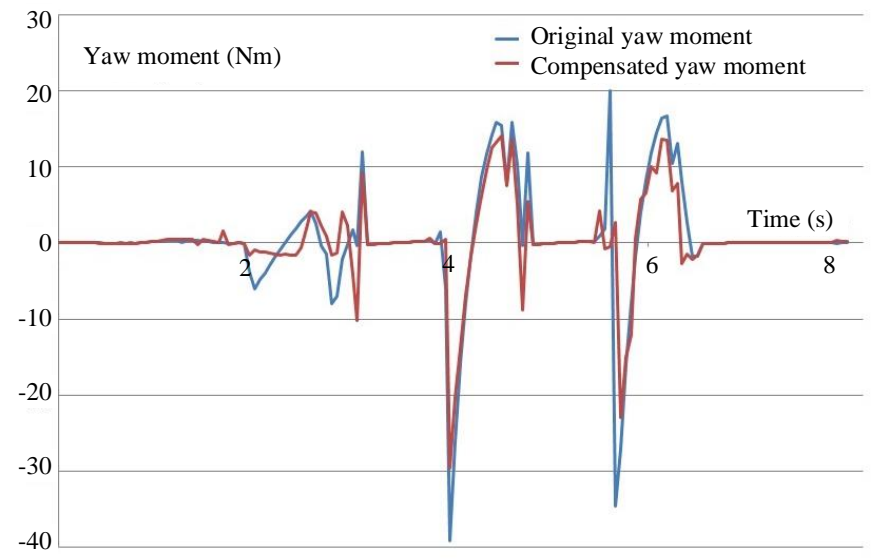

Figure 7. Yaw moment in walking simulations. Blue line: without rotational slip prevention. Red line: with rotational slip prevention.

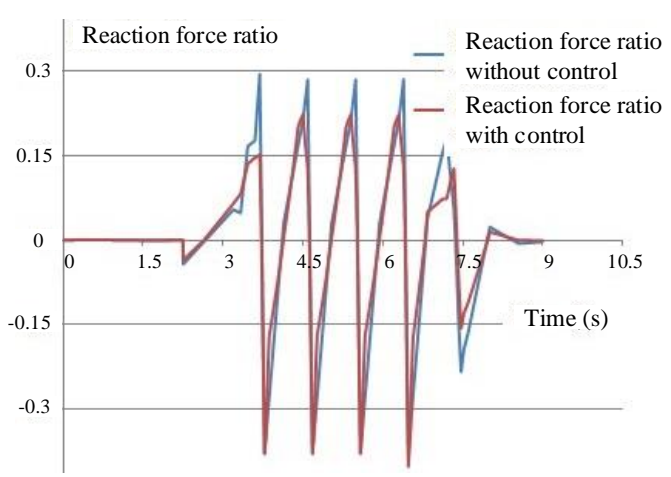

(a) Reaction force ratio.

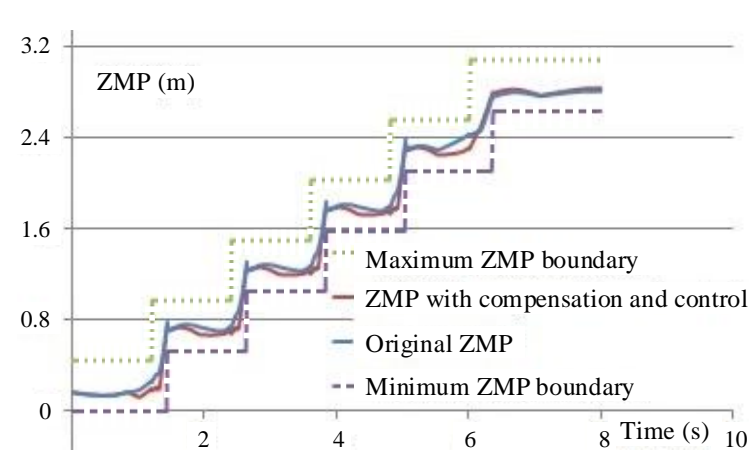

(b) ZMP position.

Figure 8. Reaction force ratio and Zero Moment Point (ZMP) in walking simulations. (a) Blue line: without proposed control. Red line: with proposed control. (b) Blue solid line: without proposed control. Red solid line: with proposed control. Short dotted line: maximum ZMP boundary. Long dotted line: minimum ZMP boundary. 


\subsection{Experiments}

The real humanoid robot BHR-5 [6] is used for experiments. Walking parameters are the same as those in simulations. The material of the ground on which the robot walked is a kind of rubber. The coefficient of static friction between the foot sole and the ground surface is estimated as 0.5 and the threshold of the coefficient of friction, $\mu_{0}$, is set to 0.3 .

Figure 9 shows the snapshots of biped walking in the real world. The yellow line (mark line) is along the walking direction in the sagittal plane. Without the proposed slip prevention methods, the robot is getting closer to the mark line during the walking as Figure 9a shows. However, once the proposed slip prevention methods is used, the robot can keep straight walking along the make line, as Figure $9 \mathrm{~b}$ shows. Hence, the effectiveness of the proposed slip prevention methods is validated.

Figure 10a illustrates the comparison of the total yaw moment with and without the proposed slip prevention methods. The blue line is the original yaw moment without control, while the red line is the compensated yaw moment with control. The maximum yaw moment of the red line is smaller than that of the blue line, which means the proposed human-inspired yaw moment compensation method effectively reduces the yaw moment. Therefore, the rotational slip is prevented by the proposed walking pattern generator in Section 3.

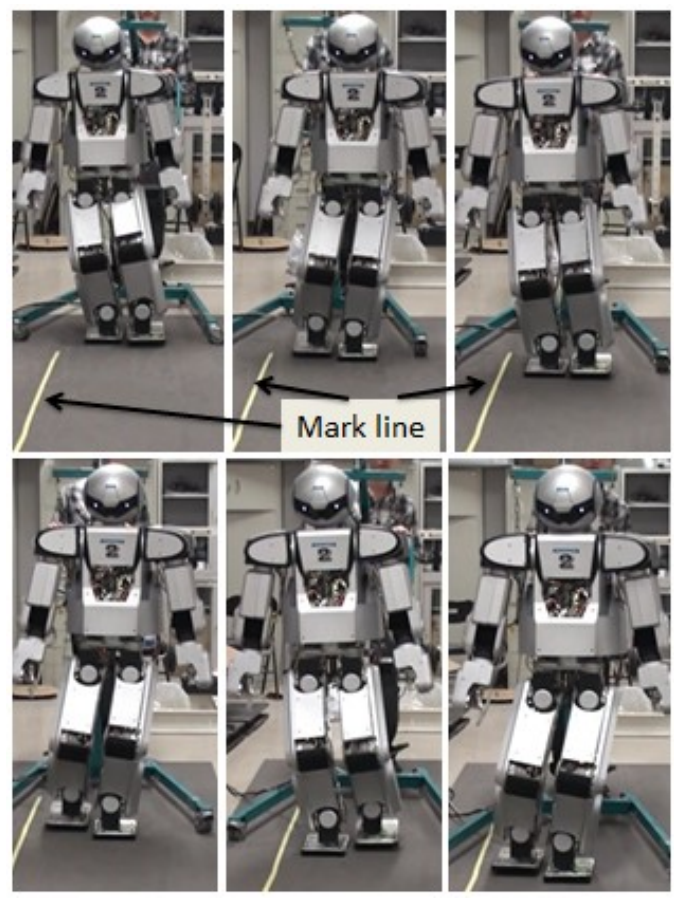

(a) Walking experiment without slip prevention method.

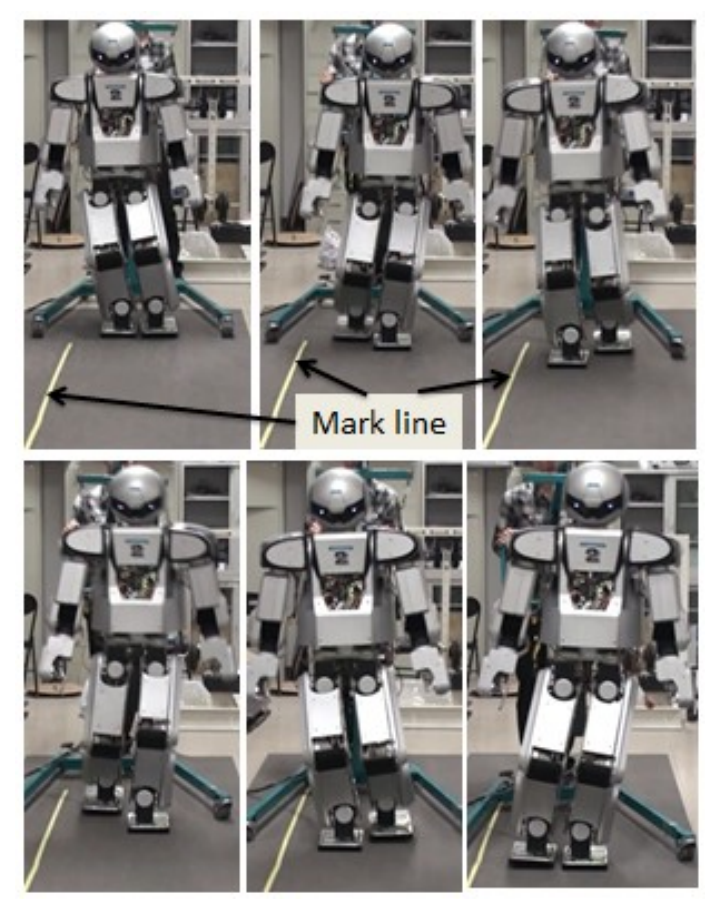

(b) Walking experiment with slip prevention method.

Figure 9. Humanoid robot walking experiments. 


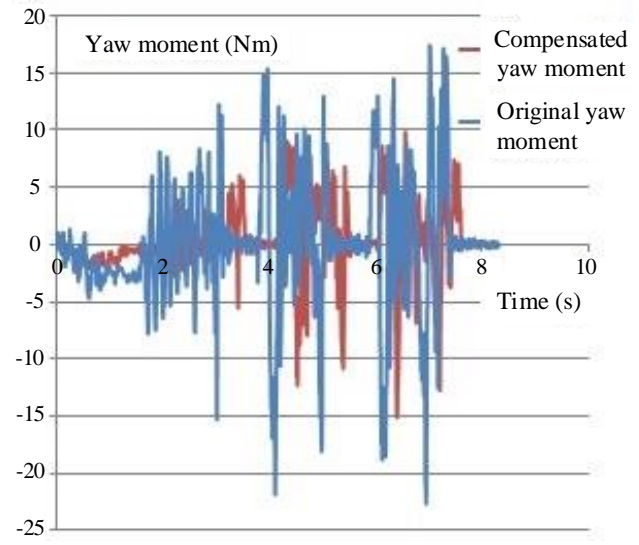

(a) Yaw moment in humanoid walking.

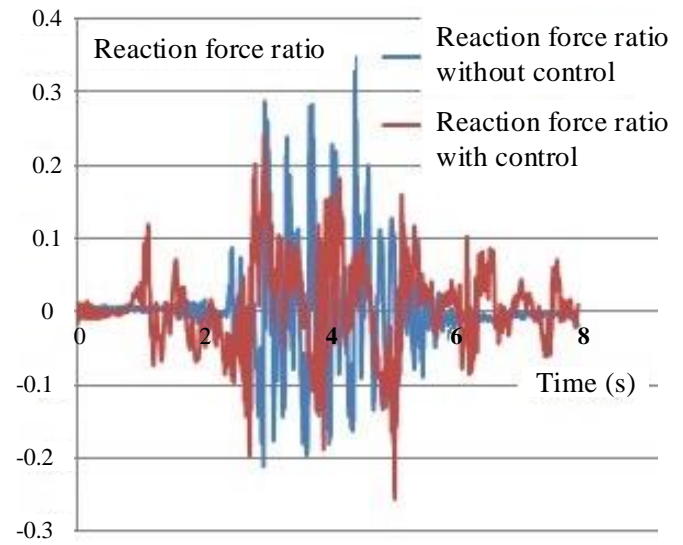

(b) Reaction force ratio in humanoid walking.

Figure 10. Yaw moment and reaction force ratio in humanoid walking. Blue lines are yaw moment and reaction force ratio without proposed control. Red lines are yaw moment and reaction force ratio with proposed control.

Figure $10 \mathrm{~b}$ compares the reaction force ratio $F_{x} / F_{z}$ during walking. It is obvious that the reaction force ratio with the proposed slip prevention methods is smaller than that without the proposed slip prevention methods. The reduced amplitudes of the red line indicates that the proposed CoM acceleration compensation method in this paper is effective in suppressing the reaction force ratio. Thus, the translational slip is prevented by the proposed reaction force ratio reduction control in Section 4 .

\section{Conclusions}

Humanoid robots may encounter slip while walking in a real environment. Even a slight slip can make a robot walk unstably or even fall. As such, a humanoid robot must be able to avoid slipping.

This paper proposes a method of preventing rotational and translational slip simultaneously for a humanoid robot. A three mass model, which takes into account the effect of swing leg on robot slip, is utilized for slip prevention. As for rotational slip prevention, a bionic walking pattern generator including arm swing and waist rotation is proposed to reduce the inertial moments in the yaw direction. This rotational slip prevention method studies from the yaw moment compensation mechanism of human walking. As for translational slip prevention, a novel reaction force ratio reduction control including compensating CoM acceleration is proposed to reduce the ratio of tangential force to normal reaction force. When the ratio of tangential force to normal reaction force is smaller than the coefficient of the ground friction, the translational slip is prevented. The simulations and experiments confirm that the humanoid robot can avoid both rotational and translational slip with the proposed slip prevention strategy.

Future work will consist in carrying out more experiments on various coefficients of friction between the foot sole and the ground surface and studying the slip prevention strategy in a more complex environment, such as slopes and soft and uneven ground.

Author Contributions: For this research article, S.Z., Q.Z. and Z.Y. conceived the idea; Z.Y. and X.C. performed the experiments; M.Q. and W.Z. analyzed the data; Q.Z. and S.Z. wrote the paper; Q.Z., Z.Y. and Q.H. reviewed and edited the paper.

Funding: This work was supported in part by the National Natural Science Foundation of China under Grant 61533004, Grant 61320106012, and Grant 61703043.

Conflicts of Interest: The authors declare no conflict of interest. 


\section{References}

1. Kuindersma, S.; Deits, R.; Fallon, M.; Valenzuela, A.; Dai, H.; Permenter, F.; Koolen, T.; Marion, P.; Tedrake, R. Optimization-based locomotion planning, estimation, and control design for the atlas humanoid robot. Auton. Robot. 2016, 40, 429-455. [CrossRef]

2. Chen, X.; Yu, Z.; Zhang, W.; Zheng, Y.; Huang, Q.; Ming, A. Bioinspired Control of Walking With Toe-Off, Heel-Strike, and Disturbance Rejection for a Biped Robot. IEEE Trans. Ind. Electron. 2017, 64, 7962-7971. [CrossRef]

3. Kajita, S.; Benallegue, M.; Cisneros, R.; Sakaguchi, T.; Nakaoka, S.I.; Morisawa, M.; Kaneko, K.; Kanehiro, F. Biped walking pattern generation based on spatially quantized dynamics. In Proceedings of the 2017 IEEE-RAS 17th International Conference on Humanoid Robotics (Humanoids), Birmingham, UK, 15-17 November 2017; pp. 599-605.

4. Koolen, T.; Bertrand, S.; Thomas, G.; De Boer, T.; Wu, T.; Smith, J.; Englsberger, J.; Pratt, J. Design of a momentum-based control framework and application to the humanoid robot atlas. Int. J. Hum. Robot. 2016, 13, 1650007. [CrossRef]

5. Ott, C.; Roa, M.A.; Schmidt, F.; Friedl, W.; Englsberger, J.; Burger, R.; Werner, A.; Dietrich, A.; Leidner, D.; Henze, B.; et al. Mechanisms and Design of DLR Humanoid Robots. In Humanoid Robotics: A Reference; Goswami, A., Vadakkepat, P., Eds.; Springer: New York, NY, USA, 2017; pp. 1-26.

6. Yu, Z.; Huang, Q.; Ma, G.; Chen, X.; Zhang, W.; Li, J.; Gao, J. Design and development of the humanoid robot BHR-5. Adv. Mech. Eng. 2014, 6, 852937. [CrossRef]

7. Yang, T.; Zhang, W.; Chen, X.; Yu, Z.; Meng, L.; Huang, Q. Turning Gait Planning Method for Humanoid Robots. Appl. Sci. 2018, 8, 1257. [CrossRef]

8. Yu, Z.; Chen, X.; Huang, Q.; Zhang, W.; Meng, L.; Zhang, W.; Gao, J. Gait planning of omnidirectional walk on inclined ground for biped robots. IEEE Trans. Syst. Man Cybern. Syst. 2016, 46, 888-897. [CrossRef]

9. Jiang, X.; Chen, X.; Yu, Z.; Zhang, W.; Meng, L.; Huang, Q. Motion Planning for Bipedal Robot to Perform Jump Maneuver. Appl. Sci. 2018, 8, 139. [CrossRef]

10. Zhu, C.; Kawamura, A. What is the real frictional constraint in biped walking?-Discussion on frictional slip with rotation. In Proceedings of the 2006 IEEE/RSJ International Conference on Intelligent Robots and Systems, Beijing, China, 9-15 October 2006; pp. 5762-5768.

11. Collins, S.H.; Adamczyk, P.G.; Kuo, A.D. Dynamic arm swinging in human walking. Proc. R. Soc. B-Biol. Sci. 2009, 276, 3679-3688. [CrossRef] [PubMed]

12. Punt, M.; Bruijn, S.M.; Wittink, H.; Van Dieën, J.H. Effect of arm swing strategy on local dynamic stability of human gait. Gait Posture 2015, 41, 504-509. [CrossRef] [PubMed]

13. Ugurlu, B.; Saglia, J.A.; Tsagarakis, N.G.; Caldwell, D.G. Yaw moment compensation for bipedal robots via intrinsic angular momentum constraint. Int. J. Hum. Robot. 2012, 9, 1250033. [CrossRef]

14. Cisneros, R.; Yokoi, K.; Yoshida, E. Yaw moment compensation by using full body motion. In Proceedings of the 2014 IEEE International Conference on Mechatronics and Automation, Tianjin, China, 3-6 August 2014; pp. 119-125.

15. Khadiv, M.; Moosavian, S.A.A.; Yousefi-Koma, A.; Sadedel, M.; Mansouri, S. Optimal gait planning for humanoids with 3D structure walking on slippery surfaces. Robotica 2017, 35, 569-587. [CrossRef]

16. Brandao, M.; Hashimoto, K.; Santos-Victor, J.; Takanishi, A. Footstep Planning for Slippery and Slanted Terrain Using Human-Inspired Models. IEEE Trans. Robot. 2016, 32, 868-879. [CrossRef]

17. Vázquez, J.A.; Velasco-Villa, M. Experimental estimation of slip in the supporting point of a biped robot. J. Appl. Res. Technol. 2013, 11, 348-359. [CrossRef]

18. Hashlamon, I.; Gülhan, M.M.; Ayit, O.; Erbatur, K. A novel method for slip prediction of walking biped robots. Robotica 2017, 35, 766-786. [CrossRef]

19. Takabayashi, Y.; Ishihara, K.; Yoshioka, M.; Liang, H.; Liu, C.; Zhu, C. Frictional constraints on the sole of a biped robot when slip. In Proceedings of the 2017 IEEE/RSJ International Conference on Intelligent Robots and Systems (IROS), Vancouver, BC, Canada, 24-28 September 2017; pp. 5011-5016.

20. Bowyer, S.A.; Baena, F.R. Dynamic frictional constraints in translation and rotation. In Proceedings of the 2014 IEEE International Conference on Robotics and Automation (ICRA), Hong Kong, China, 31 May-7 June 2014; pp. 2685-2692. 
21. Li, J.; Huang, Q.; Yu, Z.; Chen, X.; Zhang, W.; Liu, H.; Gao, J.; Duo, Y. Integral acceleration generation for slip avoidance in a planar humanoid robot. IEEE/ASME. Trans. Mech. 2015, 20, 2924-2934. [CrossRef]

22. Zhang, S.; Huang, Q.; Wang, H.; Xu, W.; Ma, G.; Liu, Y.; Yu, Z. The mechanism of yaw torque compensation in the human and motion design for humanoid robots. Int. J. Adv. Robot. Syst. 2013, 10, 57. [CrossRef]

23. Huang, Q.; Yu, Z.; Zhang, W.; Duan, X.; Huang, Y.; Li, K. Generation of humanoid walking pattern based on human walking measurement. In Proceedings of the Humanoids 2008-8th IEEE-RAS International Conference on Humanoid Robots, Daejeon, Korea, 1-3 December 2008; pp. 99-104.

24. Takenaka, T.; Matsumoto, T.; Yoshiike, T. Real time motion generation and control for biped robot-3rd report: Dynamics error compensation. In Proceedings of the 2009 IEEE/RSJ International Conference on Intelligent Robots and Systems, St. Louis, MO, USA, 10-15 October 2009; pp. 1594-1600.

25. Kajita, S.; Hirukawa, H.; Harada, K.; Yokoi, K. Introduction to Humanoid Robotics; Siciliano, B., Khatib, O., Eds.; Springer: New York, NY, USA, 2014; pp. 69-79.

(c) 2018 by the authors. Licensee MDPI, Basel, Switzerland. This article is an open access article distributed under the terms and conditions of the Creative Commons Attribution (CC BY) license (http://creativecommons.org/licenses/by/4.0/). 